\title{
Antipsychotic polypharmacy: review of mechanisms, mortality and management ${ }^{\dagger}$
}

\author{
Julie Langan, ${ }^{1}$ Polash Shajahan ${ }^{2}$
}

The Psychiatrist (2010), 34, 58-62, doi: 10.1192/pb.bp.108.024257

${ }^{1}$ NHS Lanarkshire; ${ }^{2}$ University of Glasgow, NHS Lanarkshire, Bellshill Clinic

\section{Correspondence to}

Polash Shajahan (polash.shajahan@

lanarkshire.scot.nhs.uk)
Summary Antipsychotic polypharmacy is an increasingly encountered clinical scenario. This review considers the reasons behind antipsychotic polypharmacy and the patterns of its use. We also consider the evidence of effectiveness of combined therapy $v$. monotherapy and the rationale behind the potentially beneficial combinations that are used. The potential dangers of antipsychotic polypharmacy are also discussed and the limited research regarding switching from polypharmacy to monotherapy is reviewed. Some provisional recommendations regarding antipsychotic polypharmacy are proposed.

Declaration of interest P.S. has received honoraria and hospitality from various pharmaceutical firms including Bristol-Myers Squibb, AstraZeneca, Lilly and Janssen-Cilag.
Polypharmacy was first described in the psychiatric literature in 1969. ${ }^{1}$ Studies reported variable rates of concurrent antipsychotic prescription depending on the population considered. A study in Australia, examining people receiving out-patient treatment for schizophrenia, showed a $13 \%$ rate of multiple antipsychotic prescription use. $^{2}$ One Japanese study indicated that the rate of antipsychotic polypharmacy exceeded $90 \% .^{3}$ A recent study in the UK showed an intermediate rate of $30 \%{ }^{4}$ Despite its common occurrence, the evidence base behind antipsychotic polypharmacy is widely recognised as limited and its use has been considered both a 'therapeutic option' and a 'dirty little secret'.

\section{Routes to polypharmacy}

When considering the prescription of more than one antipsychotic, perceived potential clinical benefit rather than receptor theory often leads to the chosen combination. It is increasingly evident that various non-dopaminergic receptors have an important role in the clinical profile of schizophrenia - with adrenergic, glutamatergic and serotonergic receptors (in particular $5-\mathrm{HT}_{2 \mathrm{~A}}$ receptors) involved in the pathogenesis of positive and negative symptoms. It is postulated that combined therapy might help to diversify the range of receptors affected by drug treatment and thereby improve symptoms of disease and reduce recurrence rates. $^{6}$

The National Institute for Health and Clinical Excellence (NICE) has produced algorithms for the use of antipsychotics in schizophrenia. ${ }^{7}$ The guidance states that

†See editorial, pp. 41-43, and original paper, pp. 44-46, this issue. in a first presentation, concurrent antipsychotics should not be prescribed except to cover short change-over periods (i.e. cross-tapering) and recommends that a second antipsychotic could be 'cautiously' trialled in combination with clozapine in patients whose disorder is truly 'treatment resistant', following an inadequate response to adequate trials of initial antipsychotics and also clozapine. It is only in these specific clinical settings that NICE suggests dual antipsychotic prescription should occur.

However, antipsychotic polypharmacy prescription may occur in other clinical scenarios. When clinicians aim to switch their patients from one antipsychotic to another by cross-tapering, the switch may never be fully completed as symptoms stabilise or improve. This leads to reluctance on the part of clinician or patient to complete the switch and may postpone the completion of the switch indefinitely, leading to long-term antipsychotic polypharmacy.

Clinicians sometimes prescribe antipsychotic doses close to or beyond that defined as maximum by the UK British National Formulary (BNF). ${ }^{8}$ When this occurs, highdose monitoring protocols are recommended. ${ }^{9}$ Good clinical practice suggests that we avoid high-dose prescribing in order to minimise side-effects. For this reason, clinicians may trial more than one antipsychotic at lower doses, leading once again to long-term antipsychotic polypharmacy. However, polypharmacy may in itself unwittingly result in high total dose prescribing. An audit carried out by the Prescribing Observatory for Mental Health found that $80 \%$ of patients prescribed combined antipsychotics, including those prescribed them on an 'as required' basis, had a total dose above the recommended limits by the $B N F .^{10}$ It is unclear if this incidence of high total dose prescribing within polypharmacy use is representative of current practice. 
Another possible route to antipsychotic polypharmacy is that 'stable' patients with psychosis, receiving long-term antipsychotic monotherapy, may develop an acute exacerbation of psychotic symptoms, or worsening of agitation or insomnia and be prescribed an additional antipsychotic on an 'as required' or short-term basis. However, such shortterm or 'as required' prescriptions may develop into medium- or long-term adjuvant treatments, particularly if considered successful in the short term. A natural reluctance to risk a recurrence of symptoms tends to perpetuate this situation and ultimately results in long-term antipsychotic polypharmacy.

\section{Evidence of efficacy}

There are only a small number of randomised controlled trials comparing monotherapy with polypharmacy. Some studies have shown improvements in symptoms with polypharmacy. One such trial compared clozapine with combination clozapine and risperidone in the management of severe treatment-resistant schizophrenia. Forty patients were studied over a 12-week period and an improvement in negative and positive symptoms of schizophrenia without increased rates of agranulocytosis, weight gain or seizures was seen. The authors concluded that the 'combination appeared to be safe and well tolerated' while also 'improving the overall symptoms - both the positive and negative'. ${ }^{11}$ They recognised that the study was limited by its small size and short duration. A further study in treatment-resistant schizophrenia examined the combination of sulpiride and clozapine and again showed a benefit of dual therapy in a small subgroup of patients. ${ }^{12}$ This study was also of short duration (10 weeks), and although there was no increase in extrapyramidal side-effects in the subgroup receiving dual therapy, problems with hyperprolactinaemia were noted. However, the rates and severity of hyperprolactinaemia were similar to those seen in studies looking at sulpiride alone, and were therefore not thought to be related to the addition of clozapine.

Conversely, the evidence of efficacy of polypharmacy combinations in treatment-resistant schizophrenia is not all favourable. One study published in 2004 compared olanzapine monotherapy to olanzapine combined with sulpiride and found no significant difference in positive or negative symptoms. ${ }^{13}$ Interestingly, the patients receiving dual therapy showed improvement in terms of depressive symptoms as measured by the Hamilton Rating Scale for Depression. Other studies examining the combination of risperidone and clozapine showed no significant improvement in positive or negative symptoms, ${ }^{14,15}$ but did show increased rates of sedation, akathisia, hyperprolactinaemia and elevated fasting blood glucose. ${ }^{16}$ A further study looking at combination typical and atypical antipsychotic prescription found that there was little evidence of an improvement in outcome and there was a significant increase in adverse effect burden. ${ }^{17}$ Another study found that use of more than one antipsychotic was associated with an increased incidence of metabolic syndrome, although the increased incidence could not be solely related to antipsychotic usage and was also linked to clinical and demographic factors. ${ }^{18}$ These studies have all added to the uncertainty regarding the efficacy of antipsychotic polypharmacy, and the $B N F$ states that the "prescribing of more that one antipsychotic at the same time is not recommended and may constitute a hazard'. 8

A further complication when considering antipsychotic polypharmacy is that the combinations used by clinicians are highly varied. This makes direct comparison between polypharmacy groups and monotherapy groups difficult because often the number of individuals on the same combination regimens are small. The fact that polypharmacy combinations are not consistent means that when considering a 'polypharmacy' group the drug regimen is usually not standardised and so direct comparison is fundamentally flawed. Given the large number of antipsychotics that are available for prescription, the possibilities for combination prescriptions are vast and the number of trials that would be required to examine the efficacy of all possible combinations would be innumerable.

One possible route to overcome this problem would be to categorise patients on antipsychotic polypharmacy further for example into those taking two atypical agents and those taking a typical and an atypical. Interestingly, one such study looked at this and found that $36 \%$ of patients were prescribed two atypical antipsychotics, whereas the remainder $(64 \%)$ received an atypical and a typical antipsychotic. ${ }^{19}$ An audit carried out by the Prescribing Observatory for Mental Health found that $74 \%$ of people prescribed more than one antipsychotic were prescribed an atypical in combination with a typical drug. ${ }^{10}$ These findings point to the possibility that combination of a typical and an atypical antipsychotic may be the preferred polypharmacy practice.

Many experts have suggested that it would be beneficial to 'define' a more manageable number of combination therapies, which would benefit from further investigation, with some suggesting a pharmacological approach to designing combination therapy - for instance adding a stronger dopamine receptor antagonist for those who continue to experience problematic positive symptoms. ${ }^{20}$ Gibson et al examined possible antipsychotic combinations which could be beneficial based upon pharmacological factors and named four potentially beneficial combinations, namely clozapine plus olanzapine, aripiprazole plus quetiapine, quetiapine plus olanzapine, and aripiprazole plus loxapine. ${ }^{16}$ These combinations are thought to have a more potent effect on the $\mathrm{D}_{2}$ receptor than would be seen with each drug used in isolation, thus in theory improving the symptoms of schizophrenia. Other experts suggest that owing to our limited understanding of both the pathology of schizophrenia and antipsychotic pharmacology it would be beneficial to identify patients who are already receiving combination antipsychotic therapy and are doing well, and use these combinations as a baseline for randomisation.

Many authors have reviewed the literature regarding the practice of polypharmacy and have generally concluded that except in cases where an individual has failed to respond to adequate trial of monotherapies including clozapine, antipsychotic polypharmacy has little support in the medical literature, ${ }^{16}$ and the NICE guidelines reflect this. However, NICE does recognise that there 'are circumstances where patients and clinicians serendipitously 
hit upon effective combinations' ${ }^{7}$ Therefore, polypharmacy should only be considered after a failed period of monotherapy (this would usually encompass a failed trial of clozapine). Interestingly, one study examined previous clozapine prescription in those on polypharmacy and surprisingly found that only $4 \%$ had been given a trial of clozapine before being commenced on polypharmacy. ${ }^{21}$ This finding, although not reproduced elsewhere in the literature, suggests that polypharmacy is being considered earlier in a patient's management plan than expected and that it is not being reserved for truly treatment-resistant cases.

\section{Mortality and morbidity}

There has long been recognition of the increased mortality associated with schizophrenia, with some studies estimating a relative overall mortality twice that of the general population. $^{22}$ The aetiological basis of this is poorly understood and is not explained by increased suicide rates. Individuals with a diagnosis of schizophrenia are at higher risk of cardiovascular morbidity and mortality than those in the general population. ${ }^{23}$ People with schizophrenia also have higher rates of new-onset diabetes compared with a general population control group. ${ }^{24}$ Initial studies mainly concentrated on the increased risks of these individuals with regard to physical disease leading to so-called 'natural deaths'. This was thought to be partly explained by dietary and lifestyle factors - heavy smoking, obesity, excess alcohol intake, high systolic and diastolic blood pressure, low levels of exercise and low levels of education.

Another possible reason behind the higher mortality rates seen in people with schizophrenia is the antipsychotic medications themselves. Concerns regarding antipsychotic use and its association with sudden, unexpected death have long been recognised, with prolongation of the $\mathrm{QT}_{\mathrm{c}}$ interval and the risk of torsade de pointes a well-recognised phenomenon. The increased risk of sudden cardiac death has long been associated with typical antipsychotics; however, a recent study has confirmed that this increased risk is also seen with atypical drugs. ${ }^{25}$ Interestingly, the risk of death increased with increasing dose of antipsychotic (whether typical or atypical). The study concluded that 'current users of typical and atypical antipsychotic drugs had a similar dose-related incidence of increased cardiac death'. ${ }^{25}$ This finding will lead to further debate regarding the safety of antipsychotics and indeed the practice of high-dose prescribing.

The positive impact of long-term antipsychotic use on survival is relatively neglected. Tiihonen et al found that mortality rates in people with schizophrenia who were not prescribed antipsychotics were ten times higher than in those prescribed antipsychotics. ${ }^{26}$ Their study found that patients who were prescribed any antipsychotic drug had decreased mortality compared with the 'no treatment' group. Interestingly, no difference in the reduction in mortality demonstrated was seen within the individual drugs prescribed. This study subsequently concluded that one possible route to reducing mortality among young people with schizophrenia would be to target patients who did not renew their prescriptions.
Other studies have considered the complex relationship between antipsychotic use and mortality: Enger et al suggested that 'cardiovascular risk was inversely associated with intensity of use of antipsychotic drugs', ${ }^{27}$ i.e. that the mortality may not be a direct result of the antipsychotic. However, Bralet et al suggested that there was a relationship between mortality and the dose of antipsychotics prescribed, with increased mortality seen in patients prescribed higher doses. ${ }^{28}$ However, this finding was only seen in male patients.

As polypharmacy is becoming more widespread, concerns regarding its safety have been raised. Other concerns, including increased rates of side-effects, pharmacokinetic interactions, reduced adherence to complex medication regimens and increased cost, have also been raised. Difficulties also arise regarding dose adjustments that are required depending on response and side-effects. ${ }^{29}$ More importantly, the long-term effects of antipsychotic polypharmacy have not yet been fully studied and so this too is an area of growing concern. Waddington et al found that the greater the maximum number of antipsychotics given concurrently, the shorter was patient survival'. ${ }^{23}$ A more recent study added support to Waddington's findings by demonstrating 'a graded relationship between the number of neuroleptics prescribed and mortality of those with schizophrenia, ${ }^{30}$ in fact, they found that 'those prescribed three antipsychotics simultaneously were twice as likely to die as those who were prescribed only one'. ${ }^{30}$ This study added that this finding 'could not be explained by coexistent somatic disease or other risk factors known for premature death'. ${ }^{30}$ These findings have added to the growing physical health concerns regarding antipsychotic polypharmacy.

\section{Management}

There are recommendations (both biochemical and electrocardiographic) on monitoring protocols for patients who are prescribed high-dose antipsychotic therapy. ${ }^{9}$ However, despite the common occurrence of antipsychotic polypharmacy, there is no guidance regarding monitoring protocols for these patients. In clinical practice, the guidelines for high-dose prescribing are sometimes extrapolated to cases in which more than one antipsychotic is used or where psychotropic combinations with potential cardiac interactions might occur.

There is a lack of guidance on what to do when faced with patients who are taking more than one antipsychotic, in terms of dose reduction or switching. In clinical practice, this often occurs in patients who have been in continuing care mental health units for long periods. The risk of continuing to expose such individuals to the increased morbidity and mortality of antipsychotic polypharmacy $v$. the potential deterioration in mental health from reverting to monotherapy or even dose reduction needs to be considered by psychiatrists, and presents a clinical conundrum.

To counteract the increasing trend in polypharmacy prescribing in the face of inconclusive evidence, Suzuki et al examined switching from such antipsychotic use to monotherapy. ${ }^{31}$ Individuals were often taking three antipsychotic medications and had been doing so for more than 
6 months. Of the 44 patients included in the study, $22(50 \%)$ were successfully converted to monotherapy without detriment to their mental health. A further 10 patients deteriorated following monotherapy, therefore adjuvant antipsychotics were required, albeit at lower doses. The authors concluded that most cases of multiple antipsychotic use were avoidable and recommended that polypharmacy 'should not be overused and should be the exception, to be used when other therapeutic options have failed'. ${ }^{31}$ Suzuki et al also studied patients receiving high-dose antipsychotic polypharmacy without improvement in their symptoms who were switched to second-generation (atypical) antipsychotic monotherapy. ${ }^{32}$ Of the 25 individuals recruited into the study, 23 were successfully switched to monotherapy with no detriment to their mental health at the end-point of the study (12 weeks after completion of their switch) and the vast majority showed an improvement in their Global Assessment of Functioning score.

A third study from Japan examined switching 23 patients with schizophrenia who had been taking high doses of multiple antipsychotic drugs to a single atypical antipsychotic regimen; 18 patients developed 36 episodes of psychotic exacerbations during switching, but nevertheless 20 patients were successfully switched. ${ }^{33}$ Another study examined rationalising medication in patients who were on combination oral and depot antipsychotics to a single depot antipsychotic. ${ }^{34}$ This study found that switching from polypharmacy to a single, equivalent dose of depot medication alone was associated with 'unacceptable high rates of relapse'. The reasons for this were unclear; however, the authors suggested that it might be due to the 'unique pharmacokinetics of these drugs or subtle qualitative differences between them' and concluded that 'caution is necessary whenever attempts are made to rationalise polypharmacy in schizophrenic patients' ${ }^{34}$

These studies have limitations in terms of sample size and study duration and show a mixed picture in the effectiveness of switching from polypharmacy to monotherapy; nevertheless, they are examples of the few articles available in the literature and may provide a potentially useful model for future studies.

\section{Discussion}

Antipsychotic polypharmacy is a relatively common and growing practice. The evidence base for its use is strongest in severe treatment-resistant schizophrenia, and although it is applied in other clinical settings the evidence of benefit in these patients is less clear. The possible combinations of antipsychotic drug regimens are innumerable and so a guideline similar to the one seen in the report by Gibson et al regarding the more studied combinations would be beneficial to aid physician prescribing. ${ }^{16}$ This would help consolidate our understanding of combination therapy and allow a more pragmatic approach to research. Given the unclear evidence underlying polypharmacy, we suggest a number of factors that may be useful to consider.

\section{Recommendations}

When antipsychotic polypharmacy is employed, detailed notes of why it is required should be recorded, incorporating the mechanism of how this came about, the relative benefits in terms of clinical improvement seen or anticipated and the potential harm to the patient. The notes should include a record of discussion of the patient's understanding of the potential risks and benefits and the patient's views on this. In individuals who are compelled to take antipsychotic medication (usually under the auspices of mental health legislation), antipsychotic polypharmacy should be avoided to optimise adherence. The need for detailed discussions and recording is as important in patients compelled to take treatment. In the UK, a system exists for designated medical practitioners to offer a second or independent opinion on the medication treatment plan proposed by the patient's own psychiatrist.

Individuals prescribed polypharmacy should undergo rigorous physical health checks including monitoring of body mass index and blood pressure both prior to commencement of polypharmacy and during its use. Electrocardiographic monitoring, fasting lipid and glucose profiles and liver function tests should be undertaken, and any abnormalities should be rigorously acted upon.

All cases of polypharmacy should be reviewed periodically to ensure that the use of clozapine has been considered. If clozapine has been considered and discounted, there should be documented reasons as to why this is the case. There should also be documented evidence of failure to achieve disease remission with adequate trials of a range of single drugs.

When switching from one antipsychotic to another, it is important to review that the changeover has been completed and not abandoned in the middle of a crosstaper. Cross-tapering is an important mechanism of antipsychotic polypharmacy and as such polypharmacy is possible to avoid by routinely reviewing and completing the intended switch from one antipsychotic to another.

Small dose increments of the additional antipsychotic should be undertaken in a logical and cautious approach. Consideration of potential interactions with the first antipsychotic and with other psychotropic and nonpsychotropic drugs should be borne in mind. Ideally, the total dose should be kept below $100 \%$ of the daily defined maximum dosages, for example $B N F$-defined daily dosage limits.

Switching from polypharmacy back to monotherapy is possible and should be kept under consideration. Reasons for and against reverting to monotherapy should be recorded. Monotherapy should be reinstated with caution and patients should be monitored closely for signs of relapse. Completion of a switch to monotherapy may not be possible; however, good clinical practice dictates that we regularly review and minimise the potential harm from the excessive use of antipsychotic polypharmacy.

Although the long-term effects of polypharmacy prescription are currently unknown, it is a relatively common practice. It would appear that the current clinician polypharmacy preference is the combination of a typical with an atypical antipsychotic. The evidence behind polypharmacy prescribing appears to be weighted towards increased rates of mortality proportionally related to number of antipsychotics used. Despite this, the use of polypharmacy is common, suggesting the need to investigate further the worrying 
relationship between its use and mortality. It is clear that until firm evidence regarding antipsychotic polypharmacy prescription is established it will remain more art than science.

\section{About the authors}

Julie Langan works for NHS Lanarkshire and Polash Shajahan is Consultant Psychiatrist and Honorary Senior Clinical Lecturer, University of Glasgow, NHS Lanarkshire, Bellshill Clinic, Greenmoss Place, Bellshill.

\section{References}

1 Sheppard C, Collins L, Fiorentino D, Fracchia J, Merlis S. Polypharmacy in psychiatric treatment. Incidence at a state hospital. Curr Therap Res 1969; 11: 765-74.

2 Keks NA, Alston K, Hope J, Krapivensky N, Culhane C, Tanaghow A, et al. Use of antipsychosis and adjunctive medications by an inner urban community psychiatric service. Aust N Z J Psychiatry 1999; 33: 896-901.

3 Ito C, Kubota $Y$, Sato M. A prospective survey on drug choice for prescriptions for admitted patients with schizophrenia. Psychiatry Clin Neurosci 1999; 53 (suppl): S35-40.

4 Taylor M, Shajahan P, Lawrie SM. Comparing the use and discontinuation of antipsychotics in clinical practice: an observational study. J Clin Psychiatry 2008; 69: 240-5.

5 Stahl SM. Antipsychotic polypharmacy, Part 1: Therapeutic option or dirty little secret? J Clin Psychiatry 1999; 60: 425-6.

6 Freudenreich O, Goff DC. Antipsychotic combination therapy in schizophrenia. A review of efficacy and risks of current combinations. Acta Psychiatr Scand 2002; 106: 323-30.

7 National Institute for Health and Clinical Excellence. Schizophrenia: Core Interventions in the Treatment and Management of Schizophrenia in Primary and Secondary Care. Clinical Practice Algorithms and Pathways to Care. NICE, 2002.

8 British Medical Association, Royal Pharmaceutical Society. British National Formulary, March issue. BMJ Books \& Pharmaceutical Press, 2008.

9 Taylor D, Paton C, Kerwin R. The Maudsley Prescribing Guidelines (9th ed). Informa, 2007.

10 Royal College of Psychiatrists. Prescribing Observatory for Mental Health. Royal College of Psychiatrists (http://www.rcpsych.ac.uk/ T1\%20info\%20leaflet.pdf).

11 Josiassen RC, Joseph A, Kohegyi E, Stokes S, Dadvand M, Paing WW, et al. Clozapine augmented with risperidone in the treatment of schizophrenia: a randomized, double-blind, placebo-controlled trial. Am J Psychiatry 2005; 162: 130-6.

12 Shiloh R, Zemishlany Z, Aizenberg D, Radwan M, Schwartz B, DorfmanEtrog $P$, et al. Sulpiride augmentation in people with schizophrenia partially responsive to clozapine. A double-blind, placebo-controlled study. Br J Psychiatry 1997; 171: 569-73.

13 Kotler M, Stous RD, Reznik I, Shwartz S, Weizman A, Spivak B. Sulpiride augmentation of olanzapine in the management of treatment-resistant chronic schizophrenia: evidence for improvement of mood symptomatology. Int Clin Psychopharmacol 2004; 19: 23-6.

14 Honer WG, Thornton AE, Chen EY, Chan RC, Wong JO, Bergmann A, et al. Clozapine alone versus clozapine and risperidone with refractory schizophrenia. N Engl J Med 2006; 354: 472-82.

15 Yagcioclu AE, Akbdede BB, Turgut TI, Tumuklu M, Yazici MK, Alptekin K, et al. A double-blind controlled study of adjunctive treatment with risperidone in schizophrenic patients partially responsive to clozapine: efficacy and safety. J Clin Psychiatry 2005; 66: 63-72.

16 Gibson A, Patel NC, Lauriello J, Buckley P. Anti-psychotic combinations: blind step or logical? Curr Psychiatry 2008; 7: 41-8.

17 Taylor D, Mir S, Mace S, Whiskey E. Co-prescribing of atypical and typical antipsychotics - prescribing sequence and documented outcome. Psychiatr Bull 2002; 26: 170-2.

18 Correll C, Frederickson A, Kane J, Manu P. Does antipsychotic polypharmacy the risk for metabolic syndrome? Schizophr Res 2007; 89: 91-100.

19 Thompson $\mathrm{C}$. The use of high-dose antipsychotic medication. $\mathrm{Br}$ Psychiatry 1994; 164: 448-58.

20 Sernyak MJ, Rosenheck R. Clinicians' reasons for antipsychotic coprescribing. J Clin Psychiatry 2004; 65: 1597-600.

21 Miller AL, Craig CS. Combination antipsychotics: pros, cons, and questions. Schizophr Bull 2002; 28: 105-9.

22 Tapp A, Wood AE, Secrest L, Erdmann J, Cubberley L, Kilzieh N Combination antipsychotic therapy in clinical practice. Psychiatr Serv 2003; 54: 55-9.

23 Waddington JL, Yousseff HA, Kinsella A. Mortality in schizophrenia. Antipsychotic polypharmacy and absence of adjunctive anticholinergics over the course of a 10-year prospective study. Br J Psychiatry 1998; 173: 325-9.

24 Curkendall SM, Mo J, Glasser DB, Rose Stang M, Jones JK. Cardiovascular disease in patients with schizophrenia in Saskatchewan, Canada. J Clin Psychiatry 2004; 65: 715-20.

25 Ray W, Chung C, Murray KT, Hall K, Stein M. Atypical antipsychotic drugs and the risk of sudden cardiac death. N Engl J Med 2009; 360: 225-35.

26 Tiihonen J, Walhbeck K, Lonnqvist J, Klaukka T, loannidis JP, Volavkan J, et al. Effectiveness of antipsychotic treatments in a nationwide cohort of patients in community care after first hospitalisation due to schizophrenia and schizoaffective disorder: observational follow up study. BMJ 2006; 333: 224-30.

27 Enger C, Weatherby L, Reynolds RF, Glasser DB, Walker AM. Serious cardiovascular events and mortality among patients with schizophrenia. J Nerv Ment Dis 2004; 192: 19-27.

28 Bralet MC, Yon V, Loas G, Noisette C. Cause of mortality in schizophrenic patients: prospective study of years of a cohort of 150 chronic schizophrenic patients. Encephale 2000; 26: 32-41.

29 Chen A. Noncompliance in community psychiatry: a review of clinical interventions. Hosp Community Psychiatry 1991; 42: 282-7.

30 Joukamaa M, Heliövaara M, Knekt P, Aromaa A, Raitasalo R, Lehtinen V. Schizophrenia, neuroleptic medication and mortality. $\mathrm{Br} J$ Psychiatry 2006; 188: 122-7.

31 Suzuki T, Uchida $H$, Tanaka $K$, Nomura $K$, Takano $H$, Tanabe $A$, et al. Revising polypharmacy to a single antipsychotic regimen for patients with chronic schizophrenia. Int J Neuropsychopharmacol 2004; 7: 133-42.

32 Suzuki T, Uchida H, Watanabe K, Yagi G, Kashima H. A clinical case series of switching from antipsychotic polypharmacy to monotherapy with a second-generation agent on patients with chronic schizophrenia. Prog Neuropsychopharmacol Biol Psychiatry 2004; 28: 361-9.

33 Kawai NA, Yamakawa YB, Matsuzaka HA, Baba HA, Tachikawa AC, Hori $H D$, et al. Switching from high-dose of multiple neuroleptic treatments to the single atypical neuroleptic regimen (the second report): What are the risks for psychotic exacerbations? Int Clin Psychopharmacology 2005; 20: A15.

34 Soni SD, Sampath G, Shah A, Krska J. Rationalizing neuroleptic polypharmacy in chronic schizophrenics: effects of changing to a single depot preparation. Acta Psychiatr Scand 1992; 85: 354-9. 